\title{
Health-related behaviors and multiple chronic health conditions among persons with traumatic spinal cord injury
}

\author{
Yue Cao $^{1} \cdot$ Melinda Jarnecke $^{1} \cdot$ James S. Krause $^{1}$
}

Received: 30 May 2018 / Revised: 26 October 2018 / Accepted: 30 October 2018 / Published online: 20 December 2018

(c) International Spinal Cord Society 2018

\begin{abstract}
Study design Cross-sectional study.

Objectives The purposes of this study were to assess (i) prevalence of self-reported multiple chronic conditions (MCC) in a population-based cohort of persons with traumatic spinal cord injury (TSCI) and (ii) the association between health-related behaviors and MCC.

Setting Population-based TSCI cohort.

Methods Participants included 716 adults with TSCI of at least 1-year duration who were identified through a populationbased TSCI surveillance system. Standard questions from the Behavioral Risk Factor Surveillance System measured cigarette smoking, binge drinking, planned exercises, and 10 chronic health conditions (CHC), including diabetes, heart attack, angina (or coronary artery disease), stroke, cancer, asthma, kidney disease, arthritis, depressive disorder, chronic obstructive pulmonary disease. MCC was defined as having two or more CHCs in this study. Multivariate logistic regression models were used to assess the association between health-related behaviors and MCC.

Results Almost half (45\%) of the study sample had MCC. After controlling for demographic and injury characteristics, participants with smoking history of at least 100 cigarettes were $59 \%$ more likely to develop MCC, and those who had planned exercises at least three times a week were $36 \%$ less likely to have MCC.

Conclusions We found MCC prevalence was high among people with TSCI, and MCC was associated with cigarette smoking and planned exercise.
\end{abstract}

\section{Introduction}

Multiple chronic conditions (MCC) have received prominent recent focus as an important public health issue [1]. MCC is defined as "having two or more concurrent chronic conditions that collectively have an adverse effect on health status, function, or quality of life and that require complex health care management, decision-making, or coordination," and is associated with taking more medications, seeing more than one clinician, and inconsistent health care [2]. Persons with traumatic spinal cord injury (TSCI) have an elevated risk of chronic health conditions ( $\mathrm{CHC}$ ), such as diabetes and cardiovascular disease [3-5], but the risk of

Yue Cao

caoyu@musc.edu

1 College of Health Professions, Medical University of South Carolina, Charleston, SC, USA
MCC is unknown. For those with TSCI, CHC may appear prior to injury as co-morbidities first. Then sedentary lifestyle makes such conditions progressive and ultimately exacerbates both physical health and mental health to develop additional CHC, resulting in MCC [6].

One major impacting factor for $\mathrm{CHC}$ and $\mathrm{MCC}$ is healthrelated behavior. The Alameda County Study of 1982 identified five behaviors significantly associated with preventing chronic disease. These behaviors included never smoking, regular physical activity, consuming little to no alcohol, maintaining a normal body weight, and obtaining a sufficient amount of sleep each day [7]. In the general population, cigarette smoking is a leading risk factor for the development of cardiovascular disease [8], and smoking is known to increase chronic disease and can result in premature mortality [9]. Alcohol consumption is also a known risk factor for many $\mathrm{CHC}$, including cardiovascular disease, cancer, and digestive diseases [10].

TSCI is associated with high risk behaviors, such as alcohol misuse, which contribute to the initial traumatic 
injury in some instances [11]. There is also evidence that high-risk behaviors are highly prevalent post-injury [12, 13]. One study found the prevalence for cigarette smoking in the TSCI population (35.3\%) was significantly higher than the rate of smoking in the general population [14]. Other research has also found alcohol use and binge drinking are elevated in the TSCI population at the time of injury over the general population. While alcohol use decreases after injury, the rates still remain slightly elevated [15]. Due to the loss in function that TSCI causes, those with TSCI are already predisposed to impaired physical activity, with one study finding they have $40 \%$ of the activity levels of able-bodied peers [16]. Lack of physical activity has been found to be associated with health risks, such as cardiovascular disease and type 2 diabetes [17]. In part due to impaired mobility and limited levels of physical activity, obesity prevalence in the TSCI population is also higher than the general population, with prevalence ranging from $40 \%$ to $66 \%$ [18].

Although the literature suggests people with TSCI may have higher MCC prevalence and health-related behaviors are associated with MCC, little empirical research has focused on the extent of MCC among persons with TSCI. This study attempts to identify the prevalence of MCC and assess the association between health-related behaviors and MCC in a population-based cohort with TSCI.

\section{Methods}

\section{Participants}

All participants were recruited from a subset of individuals identified through the Spinal Cord Injury Surveillance System Registry (SCISSR) in the state of South Carolina in the United States. The South Carolina SCISSR is a population-based registry of TSCI in the state. All 62 acute care non-federal hospitals in South Carolina have a statutory requirement to report uniform billing discharge data to the State Budget and Control Board. The preliminary participant pool included 1167 participants with TSCI who had previously been enrolled in a more detailed outcomes database than what could be abstracted through SCISSR existing records. Other studies also recruit participants from the same outcomes database, and it is possible some of the participants overlap. However, these studies' data collection and analyses are independent from each other (i.e., these data have never been used in a previous publication). The current study was specifically designed to measure CHC. All participants in this study met the following selection criteria: (a) minimum of 18 years of age, (b) residual impairment (excludes those with complete recovery), (c) minimum of one year since TSCI onset, and (d) resident of
South Carolina. Among the initial pool of 1167, 68 had died and 28 were deemed not eligible. Of the remaining 1071 potential participants, 155 were lost to follow-up, leaving a working sample of 916,716 of who responded $(78.2 \%$ of those who were not lost at follow-up and $66.9 \%$ including all those who were not deceased or ineligible).

\section{Procedures}

After receiving approval from an institutional review board, an introductory letter was sent describing the study and alerting potential participants of the mail-in, self-report materials to be sent $4-6$ weeks later. The non-responders received a second set of materials and a follow-up phone call. A third mailing was implemented for those who lost or misplaced materials but stated an intention to participate. Participants were offered $\$ 50$ remuneration.

\section{Measures}

Both health-related behaviors and CHC were measured by standard Behavioral Risk Factor Surveillance System (BRFSS) questions developed by the Centers for Disease Control and Prevention (CDC) available at the time of the study initiation [19]. They have since added more CHC questions. CHC questions asked participants if a doctor, nurse, or other health professional ever told them they had diabetes (not including gestational), a heart attack (also called a myocardial infarction), angina or coronary artery disease, stroke, cancer (including skin cancer and other cancers), asthma, kidney disease (not include kidney stones, bladder infection, or incontinence), arthritis, depressive disorder, chronic obstructive pulmonary disease. MCC was defined as having two or more CHC in this study.

Smoking status (yes vs. no) was measured by the BRFSS question, "have you smoked at least 100 cigarettes in your entire life?" Binge drinking (yes vs. no) was measured by "how many times during the past month did you have five or more drinks on one occasion?'. Planned exercises at least three times a week (yes vs. no) was measured by "how often do you do planned exercise? (Lifting weights, swimming, or pushing your chair for the sake of exercise. Not include passive range of motion - when somebody moves your arms or legs for you)."

Demographic characteristics included age at measurement, sex, and race/ethnicity (non-Hispanic White vs. others). Self-reported injury level was categorized as C1-4, C5-8, and other levels. Ambulatory ability (yes vs. no) was measured by asking whether a participant can walk without difficulty and without special equipment or help from another person. Years post-injury was included as another covariate. To avoid the issue of multicollinearity between age and years post-injury in the multivariate regression model, we categorized years post-injury into three groups: 
$\leq 1$ year post-injury, between 1 and 5 years post-injury, and more than 5 years post-injury. If a participant's current household income from all resources was $<\$ 20,000$, we defined it as poverty (yes vs. no) in this study. Education level was measured by a dichotomous variable (bachelor's degree and above vs. others).

\section{Analysis}

All analyses were conducted using SAS version 9.4. We presented the prevalence for MCC and each CHC. Then we ran the bivariate comparison between participants with and without MCC in terms of demographic, injury, and socioeconomic characteristics and health-related behaviors. Chi-square tests and $t$-test were used to assess the difference between two groups. We developed a multivariate logistic regression model to identify the association between MCC and health-related behaviors after controlling for participants' demographic, injury, and socioeconomic characteristics.

\section{Results}

Because one participant did not answer the CHC questions, we had 715 valid measures. Our study sample was $70 \%$ male, 56\% non-Hispanic White, and 26\% reported C1-4 injury level. Non-respondents were more likely than participants to be male (76\%), non-Hispanic White $(64 \%)$, and C1-4 injury level (34\%). Among them, $28 \%$ had no CHC, $27 \%$ had one CHC, $21 \%$ had two CHCs, $12 \%$ had three CHCs, and $12 \%$ had four or more CHCs. Based on our definition, 321 participants (45\%) had MCC. The prevalence of each individual $\mathrm{CHC}$ is presented in Table 1. Arthritis was the most prevalent condition (47\%) among participants, followed by depression (33\%).

\section{Bivariate}

Compared to participants without MCC, those having MCC were older, less likely to be male, more likely to be nonHispanic White and have C1-4 level injury. They had a higher percentage of cigarette smoking history and had low probability of regular planned exercise (Table 2).

\section{Multivariate}

In the multivariate logistic regression model (Table 3), we found older age, C1-4 level injury, and injury duration between 1 and 5 years were positively associated with the likelihood of MCC, while being male and having normal ambulatory status were associated with a reduced likelihood of MCC. Results also indicated a significant association between health-related behaviors and MCC. Participants
Table 1 Prevalence of individual chronic health conditions

\begin{tabular}{lcc}
\hline & $n(\%)$ & $95 \% \mathrm{CI}^{\mathrm{a}}$ \\
\hline Diabetes & $123(17.5)$ & $14.64-20.26$ \\
Heart attack & $53(7.5)$ & $5.52-9.39$ \\
Angina & $52(7.3)$ & $5.41-9.26$ \\
Stroke & $58(8.2)$ & $6.17-10.22$ \\
Cancer & $97(13.7)$ & $11.13-16.19$ \\
Asthma & $86(12.1)$ & $9.69-14.50$ \\
Depression & $33(32.6)$ & $29.15-36.09$ \\
COPD & $333(10.0)$ & $7.78-12.20$ \\
Kidney disease & $230(4.6)$ & $3.09-6.19$ \\
Arthritis & $71(46.7)$ & $43.03-50.38$ \\
\hline
\end{tabular}

${ }^{\mathrm{a}}$ Confidence interval

Table 2 Comparison of participants with and without MCC

\begin{tabular}{|c|c|c|c|}
\hline & $\begin{array}{l}\text { Without } \\
\text { MCC } \\
(n=394)\end{array}$ & $\begin{array}{l}\text { With MCC } \\
(n=321)\end{array}$ & $p$-Value ${ }^{\mathrm{a}}$ \\
\hline & \multicolumn{3}{|c|}{$\begin{array}{l}\% \text { (unless otherwise } \\
\text { indicated) }\end{array}$} \\
\hline $\begin{array}{l}\text { Age at measurement [mean } \\
(\mathrm{SD})]\end{array}$ & $48.55(15.50)$ & $\begin{array}{l}59.11 \\
(15.16)\end{array}$ & $<0.01$ \\
\hline $\begin{array}{l}\text { Years post-injury [mean } \\
\text { (SD)] }\end{array}$ & $10.80(8.46)$ & $\begin{array}{l}10.05 \\
(8.75)\end{array}$ & 0.27 \\
\hline Male & 76 & 63 & $<0.01$ \\
\hline Non-Hispanic White & 53 & 60 & 0.03 \\
\hline C14 injury level & 21 & 33 & $<0.01$ \\
\hline C58 injury level & 31 & 26 & 0.13 \\
\hline Walking without difficulty & 41 & 35 & 0.09 \\
\hline Income less than $20 \mathrm{~K}$ & 52 & 46 & 0.13 \\
\hline With college education & 17 & 20 & 0.37 \\
\hline $\begin{array}{l}\text { Ever smoked at least } 100 \\
\text { cigarettes }\end{array}$ & 53 & 63 & 0.01 \\
\hline Binge drinking last month & 27 & 24 & 0.35 \\
\hline $\begin{array}{l}\text { Planned exercises three } \\
\text { times a week }\end{array}$ & 28 & 20 & 0.02 \\
\hline
\end{tabular}

${ }^{\mathrm{a}}$ Chi-square tests were used for categorical variables, and $t$-test for continuous variable

with a smoking history of at least 100 cigarettes showed a $59 \%$ increase in the odds of MCC $(\mathrm{OR}=1.59)$, while those who had planned exercises at least three times a week were $36 \%(\mathrm{OR}=0.64)$ less likely to have MCC. Binge drinking was not significantly associated with MCC.

\section{Discussion}

Our study fills a gap in the literature by identifying the prevalence of MCC based on a population-based TSCI cohort, derived from a statewide TSCI registry system. This minimizes bias related to treatment factors, such as access to care, 
Table 3 Multivariate logistic regression model for MCC

\begin{tabular}{lllr}
\hline & Odds ratio & $95 \% \mathrm{CI}^{\mathrm{a}}$ & $p$-Value \\
\hline Age at measurement & 1.05 & $1.04-1.06$ & $<0.01$ \\
Male (ref = female) & 0.44 & $0.29-0.66$ & $<0.01$ \\
Non-Hispanic White (ref = others) & 1.22 & $0.83-1.80$ & 0.31 \\
$\leq 1$ year post-injury (ref = more than 5 years) & 2.14 & $0.27-16.86$ & 0.47 \\
1-5 years post-injury (ref = more than 5 years) & 1.81 & $1.22-2.67$ & $<0.01$ \\
C14 injury level (ref = others) & 1.77 & $1.14-2.74$ & 0.01 \\
C58 injury level (ref $=$ others) & 0.89 & $0.58-1.37$ & 0.60 \\
Walking without difficulty (ref $=$ others) & 0.62 & $0.43-0.91$ & 0.01 \\
Income less than 20K (ref $=$ others) & 0.98 & $0.66-1.45$ & 0.92 \\
With college education (ref $=$ others) & 1.13 & $0.70-1.83$ & 0.63 \\
Ever smoked at least 100 cigarettes $($ ref $=$ no) & 1.59 & $1.09-2.32$ & 0.02 \\
Binge drinking last month (ref $=$ no) & 1.24 & $0.82-1.88$ & 0.31 \\
Planned exercises three times a week $($ ref $=$ no) & 0.64 & $0.42-0.97$ & 0.03 \\
\hline
\end{tabular}

${ }^{\mathrm{a} C}$ Confidence Interval

within the geographic region. Our study showed $45 \%$ of participants with TSCI have MCC, while a recent study found only $26 \%$ adults have MCC in the general population [20]. Although the general population study used a slightly different $\mathrm{CHC}$ scheme from ours (adding hypertension and hepatitis, but no heart attack and depressive disorder), both studies use the same number of CHC $(n=10)$ and same definition of MCC ( $\geq 2 \mathrm{CHCs})$. The definition and the list of "chronic diseases" varied in the literature depending on the data used and researchers' academic discipline, so our findings are relative to the definitions we used to define chronic disease [21]. The BRFSS instrument we used was developed by the CDC, which has some overlapping in terms of organ systems and pathophysiology. For instance, heart attack and angina are $\mathrm{CHCs}$ of the cardiovascular system. Consequently, it is possible that the prevalence of MCC might be overestimated by using the BRFSS list. While further study is needed on the extent to which MCC in persons with SCI is elevated relative to the general population, the absolute prevalence indicates significant concern regarding the negative health outcomes associated with TSCI, even with the possibility of overestimation. While those with TSCI are living longer than ever before due to advances in health care, the higher prevalence of MCC could be a more serious health problem in the future for long-term survivors of TSCI. Our population-based study found significantly higher prevalence of $\mathrm{CHC}$ and MCC than that of one previous study, which drew its TSCI sample from a specialty rehabilitation hospital [6]. It indicated the CHC and MCC problems among TSCI population might be more serious than we thought based on our clinical experience.

It is not surprising to find older age is associated with MCC risk. Controlling for aging effect, participants with 1-5 years injury duration had higher odds of MCC than those injured more than 5 years. It might suggest a non-linear relationship between injury duration and MCC. Another possible explanation is participants with MCC are less likely to have survived more than 5 years. Females were more likely to have MCC than males, predominantly because of having a much higher prevalence of depression $(43 \%$ vs. $28 \%$ ) and arthritis (58\% vs. $42 \%)$. These are the most prevalent conditions among people with TSCI. The sex differences in depression and arthritis are consistent with the findings in the general population [22-24]. Our study finds a relationship between MCC and individual health-related behaviors. Cigarette smoking and planned exercise were significantly associated with MCC after controlling for demographic, injury, and socioeconomic variables. These findings suggest we can use health-related behavior patterns as an early indicator of MCC and to develop targeted prevention strategies. We did not find a significant association between binge drinking and MCC. One possible reason is the decrease of binge drinking prevalence from $43 \%$ preinjury to $17 \%$ post-injury [15]. Our study also only measured the post-injury drinking pattern in the past 30 days, which may not be strongly associated with MCC.

\section{Limitations}

The measurement of MCC was based on the number of $\mathrm{CHC}$, but we did not measure the timing of $\mathrm{CHC}$ in relation to TSCI. Therefore, we cannot differentiate whether they occurred before or after TSCI onset. Second, self-report information may be subject to recall bias. Third, the significant non-response rate may restrict the representativeness of the study and result in the possibility of selection bias. The selective participation could affect the overall prevalence estimate of $\mathrm{MCC}$, and the strength of the relationship between the predictors and $\mathrm{MCC}$, in either direction. Fourth, our sample only included participants from nonfederal civilian hospitals, and the results may not be generalized to the non-civilian population. Therefore, the 
findings apply to those not treated in military facilities so they have unknown generalizability to this population. Fifth, although we used standard CDC-developed questions from the BRFSS, the number of CHC assessed has been expanded since the initiation of the study. Adding more potential CHC to the equation would result in an even higher rate of MCC. Lastly, the study was cross-sectional in design and precluded any assessment of a causal relationship.

\section{Conclusion}

Our study identified a higher MCC prevalence among people with TSCI and the association of MCC with cigarette smoking and planned exercise. Health care providers need to be aware of the risk of MCC and do early screenings for MCC in addition to regular maintenance related to TSCI. Prevention is always preferable to treatment, and prevention of MCC through health-related behaviors may have a significant longterm impact on the quality of life of those with TSCI.

Acknowledgements The contents of this publication were developed under a grant from the National Institute on Disability, Independent Living, and Rehabilitation Research (NIDILRR grant number 90IF0070). NIDILRR is a Center within the Administration for Community Living (ACL), Department of Health and Human Services (HHS). The contents of this publication do not necessarily represent the policy of NIDILRR, ACL, HHS, and you should not assume endorsement by the Federal Government.

Author contributions YC was responsible for introduction, research method, and result sections. MJ was responsible for literature search and review. JK was responsible for discussion and conclusion sections.

\section{Compliance with ethical standards}

Statement of ethics We certify that all applicable institutional and governmental regulations concerning the ethical use of human volunteers were followed during the course of this research.

Conflict of interest The authors declare that they have no conflict of interest.

\section{References}

1. Center for Medicare and Medicaid Services. Chronic conditions among medicare beneficiaries, Chartbook. 2012 ed. Baltimore, MD: Center for Medicare and Medicaid Services; 2012.

2. National Quality Forum. Multiple chronic conditions (MCC) measurement framework; 2012. http://www.qualityforum.org/Projects/ Multiple_Chronic_Conditions_Measurement_Framework.aspx.

3. Charlifue SW, Weitzenkamp DA, Whiteneck GG. Longitudinal outcomes in spinal cord injury: aging, secondary conditions, and well-being. Arch Phys Med Rehabil. 1999;80:1429-34.

4. Garshick E, Kelley A, Cohen SA, Garrison A, Tun CG, Gagnon $\mathrm{D}$, et al. A prospective assessment of mortality in chronic spinal cord injury. Spinal Cord. 2005;43:408-16.

5. Krause JS, Carter RE, Pickelsimer E, Wilson D. A prospective study of health and risk of mortality after spinal cord injury. Arch Phys Med Rehabil. 2008;89:1482-91.

6. Saunders LL, Clarke A, Tate DG, Forchheimer M, Krause JS. Lifetime prevalence of chronic health conditions among persons with spinal cord injury. Arch Phys Med Rehabil. 2015;96:673-9.

7. Wingard DL, Berkman LF, Brand RJ. A multivariate analysis of health-related practices: a nine-year mortality follow-up of the Alameda County Study. Am J Epidemiol. 1982;116:765-75.

8. Steinberg MB, Schmelzer AC, Lin PN, Garcia G. Smoking as a chronic disease. Curr Cardio Risk Rep. 2010;4:413.

9. Buttar HS, Li T, Ravi N. Prevention of cardiovascular diseases: role of exercise, dietary interventions, obesity and smoking cessation. Exp Clin Cardiol. 2005;10:229-49.

10. Shield KD, Parry C, Rehm J. Chronic diseases and conditions related to alcohol use. Alcohol Use: Curr Rev. 2014;35:155-71.

11. Price C, Makintubee S, Herndon W, Istre GR. Epidemiology of traumatic spinal cord injury and acute hospitalization and rehabilitation charges for spinal cord injuries in Oklahoma, 19881990. Am J Epidemiol. 1994;139:37-47.

12. Krause JS, Coker J, Charlifue S, Whiteneck GG. Health behaviors among American Indians with spinal cord injury: comparison with data from the 1996 Behavioral Risk Factor Surveillance System. Arch Phys Med Rehabil. 1999;80:1435-40.

13. Saunders LL, Ekoja E, Whitlock CS, Dipiro ND, Gregory-Bass R, Krause JS. A comparison of health behaviors between African Americans with spinal cord injury and those in the general population. NeuroRehabilitation. 2013;33:449-56.

14. Saunders LL, Krause JS, Saladin M, Carpenter MJ. Prevalence of cigarette smoking and attempts to quit in a population-based cohort with spinal cord injury. Spinal Cord. 2015;53:641-55.

15. Davis JF, Cao Y, Krause JS. Changes in alcohol use after the onset of spinal cord injury. J Spinal Cord Med. 2018;41:230-7.

16. van den Berg-Emons RJ, Bussmann JB, Stam HJ. Accelerometrybased activity spectrum in persons with chronic physical conditions. Arch Phys Med Rehabil. 2010;91:1856-61.

17. Blair SN, Brodney S. Effects of physical inactivity and obesity on morbidity and mortality: current evidence and research issues. Med Sci Sports Exerc. 1999;31(11 Suppl):S646-62.

18. Rajan S, McNeely MJ, Warms C, Goldstein B. Clinical assessment and management of obesity in individuals with spinal cord injury: a review. J Spinal Cord Med. 2008;31:361-72.

19. CDC. Behavioral risk factor surveillance system; 2010. http://www.cdc.gov/brfss/questionnaires/pdf-ques/2010brfss.pdf.

20. Ward BW, Schiller JS, Goodman RA. Multiple chronic conditions among US adults: a 2012 update. Prev Chronic Dis. 2014;11:E62.

21. Bernell S, Howard SW. Use your words carefully: What is a chronic disease? Front Public Health. 2016;4:159.

22. Albert PR. Why is depression more prevalent in women? J Psychiatr Neurosci. 2015;40:219-21.

23. Srikanth VK, Fryer JL, Zhai G, Winzenberg TM, Hosmer D, Jones G. A meta-analysis of sex differences prevalence, incidence and severity of osteoarthritis. Osteoarthr Cartil/Osteoarthr Res Soc. 2005;13:769-81.

24. van Vollenhoven RF. Sex differences in rheumatoid arthritis: more than meets the eye. BMC Med. 2009;7:12. 